\title{
Conocimiento de los médicos generales sobre complicaciones obstétricas asociadas a riesgo cardiovascular subsecuente
}

\author{
Mario I. Lumbreras-Márquez, ${ }^{*}$ Marcelo A. de la Torre-León, ${ }^{2}$ José L. Hernández-Reguero, ${ }^{2}$ \\ Louise E. Wilkins-Haug y Ellen W. Seely ${ }^{3}$ \\ ${ }^{1}$ Harvard Medical School, Brigham \& Women's Hospital, Departamento de Ginecología y Obstetricia, Boston, Estados Unidos; ${ }^{2}$ Hospital Materno \\ Celaya, Guanajuato, México; ${ }^{3}$ Harvard Medical School, Brigham \& Women's Hospital, División de Endocrinología, Diabetes e Hipertensión, Boston, \\ Estados Unidos
}

\section{Resumen}

Introducción: La enfermedad cardiovascular (ECV) constituye la principal causa de mortalidad en mujeres; la preeclampsia (PE) y la diabetes mellitus gestacional (DMG) están asociadas a incremento en el riesgo de ECV. Objetivo: Evaluar el conocimiento de los médicos generales (MG) sobre complicaciones obstétricas asociadas a ECV. Métodos: Se envió a los MG un cuestionario electrónico anónimo basado en casos, diseñado para evaluar el entendimiento de la influencia de la historia obstétrica en el riesgo cardiovascular a largo plazo y el conocimiento general sobre riesgo de ECV. Resultados: La tasa de respuesta fue de $35 \%$ (161/465). Los participantes reconocieron que la PE y la DMG son factores de riesgo para ECV (98 y $83 \%$, respectivamente) y reportaron las siguientes estrategias de tamizaje de ECV en mujeres con historial de PE y DMG: monitoreo de presión arterial (PE $100 \%$, DMG $46 \%$ ), cálculo de índice de masa corporal (PE 68 \%, DMG 57 \%), evaluación del perfil de lípidos (PE $71 \%$, DMG $57 \%$ ), hemoglobina glucosilada (PE $26 \%$, DMG 92 \%) y glucosa en ayuno (PE 28 \%, DMG 91 \%). Conclusión: Las estrategias de tamizaje para identificar ECV en mujeres con antecedentes de PE y DMG reportadas por los MG fueron variables.

PALABRAS CLAVE: Enfermedad cardiovascular. Educación médica. Salud de la mujer. Preeclampsia. Diabetes mellitus gestacional.

\section{General practitioners' knowledge about pregnancy complications associated with long-term cardiovascular risk}

\begin{abstract}
Introduction: Cardiovascular disease (CVD) is the leading cause of mortality in women; preeclampsia (PE) and gestational diabetes mellitus (GDM) are associated with an increased risk of CVD. Objective: To evaluate general practitioners (GP) knowledge about complicated pregnancies and their association with CVD. Methods: An anonymous case-based electronic questionnaire designed to assess the level of understanding on the influence of a history of pregnancy complications on longterm cardiovascular risk and general knowledge about CVD risk was sent to GPs. Results: The response rate was $35 \%$ (161/465). The participants recognized that PE and GDM are risk factors for CVD (98 and $83 \%$, respectively), and reported the following CVD screening strategies in women with a history of PE and GDM: blood pressure monitoring (PE $100 \%$, GDM $46 \%$ ), body mass index calculation (PE $68 \%$, GDM $57 \%$ ), lipid profile evaluation (PE $71 \%$, GDM $57 \%$ ), glycated hemoglobin (PE $26 \%$, GDM $92 \%$ ), and fasting glucose (PE $28 \%$, GDM $91 \%$ ). Conclusion: GP-reported screening strategies to identify CVD in women with a history of PE and GDM were variable.
\end{abstract}

KEY WORDS: Cardiovascular disease. Medical education. Women's health. Preeclampsia. Gestational diabetes mellitus.

Correspondencia:

*Mario I. Lumbreras-Márquez

E-mail: mlumbreras-marquez@bwh.harvard.edu

0016-3813/@ 2020 Academia Nacional de Medicina de México, A.C. Publicado por Permanyer. Este es un artículo open access bajo la licencia CC BY-NC-ND (http://creativecommons.org/licenses/by-nc-nd/4.0/).
Fecha de recepción: 17-02-2020

Fecha de aceptación: 23-07-2020

DOI: $10.24875 / G M M .20000064$
Gac Med Mex. 2021;157:50-54

www.gacetamedicademexico.com
Disponible en PubMed 


\section{Introducción}

En el mundo, la enfermedad cardiovascular (ECV) es la principal causa de mortalidad en las mujeres. ${ }^{1}$ La identificación de los factores de riesgo tradicionales (por ejemplo, hipertensión [HT]), así como de aquellos que únicamente se presentan en mujeres (por ejemplo, preeclampsia $[\mathrm{PE}]$, diabetes mellitus gestacional [DMG], parto pretérmino [PPT], hijos recién nacidos con restricción del crecimiento $[\mathrm{RC}]$ ) contribuye a un mejor entendimiento de los mecanismos que propician la ECV. ${ }^{2,3}$ Una proporción significativa de mujeres tiene embarazos que se complican con PE, DMG, PPT y RC. ${ }^{4}$ La incidencia de alguna de estas complicaciones va de $2 \%$ a más de $12 \%$ y cada una está asociada a incremento en el riesgo de ECV., ${ }^{4,6-9}$

Las guías de la American Heart Association (AHA) y del American College of Obstetricians and Gynecologists incluyen la PE como un factor de riesgo para ECV; ambas organizaciones recomiendan preguntar sobre el antecedente de PE con el fin de recomendar cambios en el estilo de vida (evitar el tabaquismo, fomentar la actividad física, reducir el peso en caso necesario, además de seguir una dieta tipo DASH [dietary approaches to stop hypertension]) en las mujeres con ese antecedente clínico. ${ }^{10-14}$ Otros factores de riesgo para ECV mencionados en la guía de la AHA de 2011 incluyen DMG e HT gestacional. Además, como parte del cálculo de riesgo de ECV, los profesionales de la salud deben describir detalladamente las complicaciones obstétricas, incluidas PE, DMG, PPT y RC.12

En la guía de práctica clínica mexicana sobre trastornos hipertensivos del embarazo publicada en 2017 se recomienda que las pacientes con antecedente de PE deben recibir consejería sobre la importancia de la actividad física y la reducción de peso en caso necesario; además, se sugiere un tamizaje anual para $\mathrm{HT}$, dislipidemia y diabetes, con la finalidad de reducir el riesgo de ECV. ${ }^{15}$ La guía de práctica clínica mexicana sobre la prevención y el diagnóstico de ECV en mujeres no ha sido actualizada desde $2008 .{ }^{16}$ En ella, la $D M G$ es la única complicación obstétrica referida como factor de riesgo para ECV. Es importante mencionar que los médicos mexicanos frecuentemente utilizan las guías estadounidenses en conjunto con las mexicanas para proveer atención médica.

De acuerdo con publicaciones en países desarrollados, existen barreras para implementar estrategias que permitan mejorar el estilo de vida de las pacientes con las complicaciones obstétricas descritas, una de ellas es la falta de conocimiento de los médicos sobre la asociación entre las complicaciones obstétricas y la ECV. ${ }^{17-19}$ Sin embargo, hay información limitada en países en desarrollo como México y otros países en América Latina. En este estudio, el objetivo principal fue evaluar el conocimiento de los médicos generales en México con el fin de promover estrategias educativas que permitan la mejora de la atención de la salud de las mujeres, ya que esos profesionales de la salud proveen cuidado médico a una gran proporción de mujeres en el país. ${ }^{20}$ Se utilizó un cuestionario basado en casos $^{19}$ para evaluar el conocimiento de los médicos generales de la Secretaría de Salud del Estado de Guanajuato, México, acerca de antecedentes de complicaciones obstétricas y el riesgo de ECV subsecuente; el cuestionario está basado en la guías de la AHA de 2011.12

\section{Métodos}

El protocolo de investigación fue aprobado por el comité de investigación del Hospital Materno Celaya, Secretaría de Salud del Estado de Guanajuato, México. De octubre a noviembre de 2018, fue enviada una encuesta voluntaria, electrónica y anónima a los médicos generales registrados en la base de datos de esa Secretaría ( $N=465)$.

La encuesta se basó en una publicación previa realizada en un hospital de enseñanza en Boston, Massachusetts, Estados Unidos. ${ }^{19} \mathrm{El}$ cuestionario está enfocado en dos temas: conocimiento de los participantes sobre la influencia de los antecedentes obstétricos en el riesgo cardiovascular a largo plazo; además, se evalúa el conocimiento general sobre riesgo de ECV conforme las guías de la AHA. ${ }^{12}$ Se realizó una traducción cultural del cuestionario siguiendo las recomendaciones de la Organización Mundial de la Salud ${ }^{21}$ (traducción hacia adelante, panel de expertos para traducción inversa, preprueba, entrevistas, versión final); todos los autores participaron en este proceso para obtener la versión definitiva. La encuesta también recolectó información demográfica en la que se incluyó la experiencia profesional y las características del centro de trabajo (primer, segundo y tercer nivel de atención).

Con un enfoque similar al reportado por WilkinsHaug et al., ${ }^{19}$ las respuestas a preguntas de opción múltiple y las respuestas múltiples, incluidas las respuestas correctas e incorrectas, fueron ordenadas para obtener una escala en el conocimiento sobre los aspectos cardiovasculares: bajo, promedio 0 alto. Tomando en cuenta las respuestas de listas con cinco 
a ocho opciones, se consideró conocimiento bajo cuando se obtuvo una o ninguna respuesta correcta; conocimiento promedio, cuando $\geq 50 \%$ de las respuestas fueron correctas; y conocimiento alto, cuando se obtuvieron todas las respuestas correctas.

Se enviaron cinco invitaciones por correo electrónico con un enlace para completar el cuestionario a los médicos generales registrados en la base de datos (correos electrónicos disponibles: 465/2463, $19 \%$ ). No se envió una versión del cuestionario en papel al resto de los médicos generales por correo debido a limitaciones financieras. La encuesta fue creada y guardada en Research Electronic Data Capture (REDCap), ${ }^{22}$ recurso facilitado por el Brigham and Women's Hospital de la Harvard Medical School, en Boston, Massachusetts, Estados Unidos.

Los datos se presentaron como media \pm desviación estándar), mediana con rango intercuartílico o número y porcentaje. Para el análisis de subgrupos (por ejemplo, tipo de centro) se utilizó análisis de varianza (ANOVA) para comparar más de dos subgrupos con una variable dependiente continua. La fiabilidad y la validez del cuestionario fueron analizadas con alfa de Cronbach y análisis factorial confirmatorio. El análisis estadístico se realizó en Stata (Stata Corp, TX, Estados Unidos).

\section{Resultados}

La tasa de respuesta fue de $35 \%$ (161/465). No hubo encuestas incompletas. La experiencia profesional y la distribución de los médicos por nivel de atención se presentan en la Tabla 1; $71 \%$ de los médicos generales indicó que investigaba la historia clínica obstétrica cuando evaluaba el riesgo de ECV, $98 \%$ reconoció a la PE como un factor de riesgo para ECV, 83 \% consideró que la DMG está asociada a ECV y una proporción menor de los participantes reconoció la importancia del PPT (11\%) y la RC (22\%) como factores de riesgo para ECV. En una pregunta enfocada a la consulta anual para evaluar el riesgo de ECV, los médicos generales interrogan consistentemente a las mujeres acerca de la historia clínica obstétrica: PE $91 \%$, DMG $85 \%$, PPT $22 \%$, RC $29 \%$; solo $9 \%$ de los participantes reportó que no incorpora ninguna de estas complicaciones obstétricas al evaluar el riesgo de ECV en las mujeres. El tamiz cardiometabólico reportado por los médicos generales para pacientes con antecedente de PE y DMG se describe en la Tabla 2. Las intervenciones reportadas por los médicos evidencian variabilidad importante entre mujeres con antecedente de PE y DMG.
Tabla 1. Distribución de 161 médicos según experiencia profesional y nivel de atención

\begin{tabular}{|l|c|c|}
\hline Caracteristica & n & $\%$ \\
\hline \begin{tabular}{l|l|} 
Experiencia profesional (años) \\
$\quad 5$
\end{tabular} & & \\
\hline $5-10$ & 21 & 13 \\
\hline $11-15$ & 62 & 38 \\
\hline $16-20$ & 45 & 28 \\
\hline 20 & 17 & 11 \\
\hline $\begin{array}{l}\text { Nivel de atención } \\
\text { Primer } \\
\text { Segundo }\end{array}$ & 16 & 10 \\
\hline Tercer & & \\
\hline
\end{tabular}

Tabla 2. Evaluación cardiometabólica reportada por 161 médicos para mujeres con antecedente de preeclampsia y diabetes gestacional

\begin{tabular}{|l|c|c|c|c|}
\hline \multirow{2}{*}{ Evaluación } & \multicolumn{2}{|c|}{ Preeclampsia } & \multicolumn{2}{|c|}{$\begin{array}{c}\text { Diabetes } \\
\text { gestacional }\end{array}$} \\
\cline { 2 - 5 } & n & $\%$ & $n$ & $\%$ \\
\hline Presión arterial & 161 & 100 & 74 & 46 \\
\hline Índice de masa corporal & 109 & 68 & 91 & 57 \\
\hline Perfil de lípidos & 115 & 71 & 91 & 57 \\
\hline Hemoglobina glucosilada & 42 & 26 & 148 & 92 \\
\hline Glucosa en ayuno & 45 & 28 & 147 & 91 \\
\hline
\end{tabular}

En cuanto al conocimiento cardiovascular, $52.8 \%$ de los participantes fue considerado con nivel promedio. Respecto a la pregunta relacionada con factores de riesgo de ECV, el nivel de conocimiento fue alto en $12.4 \%$ de los participantes y bajo en $8.1 \%$. Más de $50 \%$ de los participantes identificó las siguientes estrategias educativas como recursos que les permitirían mejorar su habilidad para brindar una consejería adecuada a las mujeres respecto a ECV: congresos (81\%), recursos en línea $(80 \%)$ y guías de práctica clínica actualizadas (76\%). No hubo diferencia en la puntuación del cuestionario obtenida por los participantes cuando se realizaron análisis de subgrupos tomando en cuenta la experiencia profesional y el tipo de hospital (no se muestran los datos).

Se encontró que el cuestionario resultó ser confiable y válido; el alfa de Cronbach fue de 0.85 para las siete preguntas relacionadas con la historia clínica, estrategias de tamizaje y prevención. Para el análisis factorial confirmatorio se probó un modelo con dos factores (utilizando las preguntas del cuestionario 
original ${ }^{19}$ ): historia clínica (preguntas $\left.3,4,5,9\right)$ y estrategias de tamiz-prevención (preguntas 6, 7, 8), incluyendo los datos recolectados en el presente estudio ( $n=161)$. Las variables sobre datos demográficos y los recursos educativos fueron excluidas ya que esa información es complementaria. Se utilizó el criterio de la máxima verosimilitud; el ajuste del modelo fue adecuado con un error cuadrático medio de aproximación de 0.076, intervalo de confianza de $90 \%(0.016,0.115)$, índice de ajuste comparativo de 0.912, índice de Tucker-Lewis de 0.858 ( $\chi^{2}$ $p<0.001$ ); para las cargas factoriales se observaron coeficientes estandarizados de -0.088 a 0.749 . La correlación entre historial clínico y tamiz-prevención fue de 0.85. Un análisis exploratorio utilizando un modelo con tres factores (historia clínica, estrategias de tamizaje y estrategias de prevención) confirmó resultados similares (no se muestran los datos).

\section{Discusión}

Por décadas, la investigación sobre ECV se ha enfocado en los hombres, lo que ha conllevado a subestimación en las diferencias relacionadas con el sexo desde una perspectiva etiológica, diagnóstica, preventiva y terapéutica. Además, el manejo óptimo de la ECV pudiera diferir entre hombres y mujeres. ${ }^{2}$ El cuidado que los médicos generales proveen es crucial para evaluar el riesgo cardiovascular en las mujeres y para mejorar las estrategias preventivas en esa población. De acuerdo con información del Instituto Nacional de Estadística y Geografía, en México había 343000 médicos en 2014 (58 \% médicos generales, $24 \%$ especialistas y $18 \%$ otros especialistas). ${ }^{20}$ Tradicionalmente, los ginecólogos-obstetras son considerados los médicos de atención primaria de las mujeres durante su vida reproductiva, sin embargo, el papel de los médicos generales es crucial en este contexto ya que ellos proveen la evaluación del riesgo cardiovascular y el seguimiento a una proporción significativa de mujeres.

En este estudio reportamos los resultados de una encuesta desarrollada para evaluar el conocimiento de los médicos generales en cuanto a la influencia del historial obstétrico en la salud cardiovascular y en la evaluación del riesgo conforme las guías de la AHA de 2011 para la prevención de ECV en mujeres. Si bien es cierto que la mayoría de los médicos generales identificó la PE y la DMG como factores de riesgo para ECV, una proporción menor reconoció la importancia del PPT y la RC; además, las estrategias de tamizaje que los médicos proveen no fueron óptimas.
A lo largo del tiempo se ha incrementado el reconocimiento de la PE como factor de riesgo para ECV entre ginecólogos-obstetras e internistas. En comparación con los resultados reportados por WilkinsHaug et al..$^{19}$ en 2015, quienes identificaron que $64 \%$ de ginecólogos-obstetras e internistas estadounidenses identificaron a la PE como factor de riesgo para ECV, encontramos que en 2018 casi todos los médicos generales $(98 \%)$ reconocieron a la PE como factor de riesgo. Sin embargo, la proporción de médicos que investigó el historial obstétrico fue menor en nuestro estudio (83\% de los ginecólogos-obstetras e internistas versus $71 \%$ de los médicos generales). La proporción de médicos que solicitaron rutinariamente glucosa en ayuno en pacientes con antecedente de PE fue similar en nuestro estudio (34\% de los ginecólogos-obstetras e internistas versus $28 \%$ de los médicos generales).

En otro estudio de 2012 en Estados Unidos en el cual también se evaluó el conocimiento de los internistas y ginecólogos-obstetras, numerosos participantes no estaban seguros o no sabían si la PE era un factor de riesgo de enfermedad isquémica (56 y $23 \%$ ), de evento vascular cerebral (48 y $38 \%$ ) y de disminución en la esperanza de vida (79 y $77 \%$ ). El $9 \%$ de los internistas y $38 \%$ de los ginecólogos-obstetras reportaron que proveían asesoría para la reducción del riesgo cardiovascular en pacientes con antecedente de PE. ${ }^{18}$

En un estudio canadiense de ginecólogos-obstetras y parteras en 2007, solo $54 \%$ de los participantes sabía que las mujeres con antecedente de PE tienen un riesgo incrementado de HT en comparación con las mujeres nulíparas; ${ }^{17}$ además, $57 \%$ de los participantes reportó que proporcionaba consejería respecto al riesgo de $\mathrm{HT}$ en $50 \%$ de las ocasiones.

El conocimiento de los médicos generales respecto a las complicaciones obstétricas y la evaluación del riesgo cardiovascular han sido reportados en otras investigaciones. En un estudio en Holanda enfocado a médicos generales en 2009, los autores revisaron los expedientes de 35 mujeres con antecedente de PE e incluyeron 150 controles; encontraron que la presión arterial era medida en los primeros tres meses después del parto en $50 \%$ de las mujeres con antecedente de $\mathrm{PE}$, sin ninguna otra cita de seguimiento..$^{23}$ Resultados similares se reportaron en un análisis portugués en 2014, en el que un cuestionario sobre los cambios en el estilo de vida y las estrategias de tamiz para ECV fue enviado a mujeres con diagnóstico de PE a los seis meses o más después del parto; solo $31 \%$ de las participantes reportó que su médico general evaluó el antecedente de PE como un factor de riesgo para ECV. ${ }^{24}$ 
La investigación que aquí se presenta es uno de los primeros estudios relacionados con el conocimiento sobre la asociación entre complicaciones obstétricas y ECV subsecuente en médicos generales en América Latina, lo cual es importante ya que la incidencia, morbilidad y mortalidad varían en los diferentes países, razas y grupos étnicos. ${ }^{25,26}$ Sin embargo, nuestra investigación tiene varias limitaciones: únicamente se incluyeron médicos generales que contaban con correo electrónico y la tasa de respuesta fue relativamente baja; además, no se dispuso de información para comparar a los participantes con los médicos que no participaron.

\section{Conclusión}

Son necesarios esfuerzos educativos para mejorar el conocimiento de los médicos generales respecto a los antecedentes obstétricos y ECV subsecuente, así como a las estrategias de tamizaje indicadas en esta población. Actualmente, todos los médicos involucrados en el cuidado de la salud de la mujer, incluidos los médicos generales, deben investigar los antecedentes obstétricos para identificar a las pacientes con incremento del riesgo cardiovascular.

\section{Conflicto de intereses}

Ninguno de los autores tiene conflictos de intereses que declarar.

\section{Financiamiento}

Este estudio no recibió ninguna subvención específica de ninguna agencia de financiación en los sectores público, comercial o sin fines de lucro.

\section{Responsabilidades éticas}

Protección de sujetos humanos y animales. Los autores declaran que los procedimientos seguidos estaban de acuerdo con las regulaciones del comité de ética de investigación clínica relevante y con los del Código de Ética de la Asociación Médica Mundial (Declaración de Helsinki).

Confidencialidad de los datos. Los autores declaran que no aparecen datos de pacientes en este artículo.

Derecho a la privacidad y consentimiento informado. Los autores declaran que no aparecen datos de pacientes en este artículo.

\section{Bibliografía}

1. The Global Health Observatory. 10 leading causes of death in females. [Internet]. World Health Organization; 2019.

2. Garcia M, Mulvagh SL, Merz CN, Buring JE, Manson JE. Cardiovascular disease in women: clinical perspectives. Circ Res. 2016;118:1273-1293.

3. Hauspurg A, Ying W, Hubel CA, Michos ED, Ouyang P. Adverse pregnancy outcomes and future maternal cardiovascular disease. Clin Cardiol. 2018;41:239-246.

4. Rich-Edwards JW, Fraser A, Lawlor DA, Catov JM. Pregnancy characteristics and women's future cardiovascular health: an underused opportunity to improve women's health? Epidemiol Rev. 2014;36(1):57-70.

5. Fraser A, Nelson SM, MacDonald-Wallis C, Cherry L, Butler E, Sattar N, et al. Associations of pregnancy complications with calculated cardiovascular disease risk and cardiovascular risk factors in middle age: the Avon Longitudinal Study of Parents and Children. Circulation. 2012;125:1367-1380.

6. Bellamy L, Casas JP, Hingorani AD, Williams DJ. Pre-eclampsia and risk of cardiovascular disease and cancer in later life: systematic review and meta-analysis. BMJ. 2007;335(7627):974.

7. Bonamy AK, Parikh NI, Cnattingius S, Ludvigsson JF, Ingelsson E. Birth characteristics and subsequent risks of maternal cardiovascular disease: effects of gestational age and fetal growth. Circulation. 2011;124(25): 2839-2846.

8. Vrachnis N, Augoulea A, Iliodromiti Z, Lambrinoudaki I, Sifakis S, Creatsas G. Previous gestational diabetes mellitus and markers of cardiovascular risk. Int J Endocrinol. 2012;2012:458610.

9. Rich-Edwards JW, Klungsoyr K, Wilcox AJ, Skjaerven R. Duration of pregnancy, even at term, predicts long-term risk of coronary heart disease and stroke mortality in women: a population-based study. Am J Obstet Gynecol. 2015;213:518.e1-518.e8.

10. Grundy SM, Stone NJ, Bailey AL, Beam C, Birtcher KK, Blumenthal RS, et al. 2018 AHA/ACC/AACVPR/AAPA/ABC/ACPM/ADA/AGS/APhA/ASPC/ NLA/PCNA Guideline on the Management of Blood Cholesterol: a report of the American College of Cardiology/American Heart Association Task Force on Clinical Practice Guidelines. J Am Coll Cardiol. 2019;73:3168-3209.

11. ACOG Practice Bulletin No. 202: gestational hypertension and preeclampsia. Obstet Gynecol. 2019;133:1.

12. Mosca L, Benjamin EJ, Berra K, Bezanson JL, Dolor RJ, Lloyd-Jones DM, et al. Effectiveness-based guidelines for the prevention of cardiovascular disease in women - 2011 update: a guideline from the American Heart Association. Circulation. 2011;123:1243-1262.

13. Bushnell C, McCullough LD, Awad IA, Chireau MV, Fedder WN, Furie KL, et al. Guidelines for the prevention of stroke in women: a statement for healthcare professionals from the American Heart Association/American Stroke Association. Stroke. 2014;45:1545-1588.

14. Hypertension in pregnancy. Report of the American College of Obstetricians and Gynecologists' Task Force on Hypertension in Pregnancy. Obstet Gynecol. 2013;122:1122-1231.

15. Prevención, diagnóstico y tratamiento de la Preeclampsia en segundo y tercer nivel de atención. México: Secretaría de Salud; 2017.

16. Prevención y diagnóstico oportuno del riesgo de enfermedad cardiovascular en la mujer en el primer nivel de atención. México: Secretaría de Salud; 2008.

17. MacDonald SE, Walker M, Ramshaw H, Godwin M, Chen XK, Smith G. Hypertensive disorders of pregnancy and long-term risk of hypertension: what do Ontario prenatal care providers know and what do they communicate? J Obs Gynaecol Can. 2007;29:705-710.

18. Young B, Hacker MR, Rana S. Physicians' knowledge of future vascular disease in women with preeclampsia. Hypertens Pregnancy. 2012;31:50-58.

19. Wilkins-Haug L, Celi A, Thomas A, Frolkis J, Seely EW. Recognition by women's health care providers of long-term cardiovascular disease risk after preeclampsia. Obstet Gynecol. 2015;125:1287-1292.

20. Estadísticas a propósito del día del médico [Internet]. México: Instituto Nacional de Estadística y Geografía; c2019.

21. Process of translation and adaptation of instruments [Internet]. World Health Organization; c2019.

22. Harris PA, Taylor R, Thielke R, Payne J, Gonzalez N, Conde JG. Research electronic data capture (REDCap)-a metadata-driven methodology and workflow process for providing translational research informatics support. J Biomed Inform. 2009;42:377-381.

23. Nijdam ME, Timmerman MR, Franx A, Bruinse HW, Numans ME, Grobbee $\mathrm{DE}$, et al. Cardiovascular risk factor assessment after pre-eclampsia in primary care. BMC Fam Pract. 2009;10:77.

24. Viana-Pinto P, Rei M, Machado AP, Montenegro N. Preeclampsia and future cardiovascular risk: are women and general practitioners aware of this relationship? The experience from a Portuguese centre. Obstet Gynecol Int. 2014;2014:53153.

25. Hedderson MM, Darbinian JA, Ferrara A. Disparities in the risk of gestational diabetes by race-ethnicity and country of birth. Paediatr Perinat Epidemiol. 2010;24:441-448.

26. Gong J, Savitz DA, Stein CR, Engel SM. Maternal ethnicity and pre-eclampsia in New York City, 1995-2003. Paediatr Perinat Epidemiol. 2012;26:45-52. 